\title{
Creating a Real-World Data, United States Healthcare Claims-Based Adaptation of Kurtzke Functional Systems Scores for Assessing Multiple Sclerosis Severity and Progression
}

\author{
Chi T. L. Truong · Hoa V. Le • Aaron W. Kamauu · John R. Holmen • \\ Christopher L. Fillmore · Monica G. Kobayashi · Schiffon L. Wong
}

Received: January 29, 2021 / Accepted: July 13, 2021 / Published online: July 31, 2021

(C) The Author(s) 2021

\begin{abstract}
Introduction: This article describes the development of a unique mapping of the Kurtzke Functional Systems Scores (KFSS) from International Classification of Diseases, 9th revision, Clinical Modification (ICD-9-CM) codes among multiple sclerosis (MS) patients within a US Integrated Delivery Network (IDN). Valid identification of increasing disability may allow deeper insight into MS progression and possible treatments.
\end{abstract}

Supplementary Information The online version contains supplementary material available at https:// doi.org/10.1007/s12325-021-01858-9.

C. T. L. Truong

MedCodeWorld, Chapel Hill, NC, USA

H. V. Le $(\bowtie)$

PAREXEL International, 2520 Meridian Pkwy \#200,

Durham, NC 27713, USA

e-mail: hoa.vanle@gmail.com

A. W. Kamauu

PAREXEL, Bountiful, UT, USA

J. R. Holmen · C. L. Fillmore

Intermountain Healthcare, Murray, UT, USA

M. G. Kobayashi

PAREXEL, Durham, NC, USA

S. L. Wong

EMD Serono, Billerica, MA, USA
Methods: This cohort study identified MS patients in the IDN, Intermountain Healthcare. Experienced clinicians and informaticists mapped electronic health record ICD-9-CM codes to KFSS components generating a modified Kurtzke Expanded Disability Status Scale (EDSS). Modified EDSS scores were used to assess disability progression by calculating means, medians, ranges, and changes in KFSS and modified EDSS scores.

Results: Overall, 608/2960 (20.5\%) patients were identified as having MS progression and presented a wide range of scores on the EDSS 10 -point scale. The median (range) first and second EDSS scores were 0 (0-6) and 5 (1-8), respectively. The median (range) change from first to second score was 5 (1-7.5). The median first KFSS score for all systems was 0 , and the mean differed among components. The highest mean first KFSS score (1.06) was measured for sensory function and lowest $(0.12)$ for cerebellar functions. Of the 544 patients with their first EDSS scores in the $\leq 2.5$ group, $75.2 \%$ and $15.1 \%$ had their second EDSS scores in group $3-5.5$ and $\geq 6$, respectively. Of the 62 patients with their first EDSS score in the 3-5.5 group, $58.1 \%$ had their second EDSS scores in group $\geq 6$.

Conclusion: This innovative mapping technique is a promising method for future comparative effectiveness and safety research of Disease-Modifying Therapy in Real-World Data repositories. Future research to validate and 
expand on this method in another healthcare database is encouraged.

Keywords: ICD-9 mapping; KFSS; EDSS; MS disability; Algorithm; Real-world data

\section{Key Summary Points}

Why carry out this study?

There is an interest in tailoring multiple sclerosis (MS) treatment based on disease subtype and level of disability.

By mapping the components of Kurtzke Functional Systems Scores (KFSS) to generate Expanded Disability Status Scale (EDSS) scores, it is possible to identify patients with MS disability progression.

Limitations of the study are noted in the Discussion.

\section{What was learned from this study?}

Mapping of KFSS makes it possible to calculate changes in EDSS score.

This study provides a promising method for the comparative effectiveness and safety of disease-modifying therapies in the treatment of MS.

\section{INTRODUCTION}

There are significant incentives to tailor multiple sclerosis (MS) pharmaceutical treatments by identifying MS patient populations and the disease-modifying therapies (DMTs) most effective for their disease subtype and progression level. As a result, the demand for observational studies in MS using real-world data has grown $[12,22]$.

Current disease assessment practices for MS patients include an evaluation of demyelination via the Kurtzke Functional Systems Status Scale (KFSS). Developed by John Kurtzke, MD, the KFSS measures demyelination impact on body systems. The KFSS components include systems related to the brain (pyramidal, cerebellar, brainstem, sensory, and cerebral) as well as three body functioning systems: bowel and bladder, visual, and motor. The KFSS is widely implemented by neurologists and general practitioners $[14,20]$.

As useful and productive as the KFSS has been in assessing MS patient functioning, US Integrated Delivery Network (IDN) administrative International Classification of Diseases, 9th revision, Clinical Modification (ICD-9-CM) codes do not exist for results of clinical assessment instruments such as the KFSS. For the MS patient population, measuring KFSS from healthcare claims databases would improve the effectiveness of DMT studies and may yield better treatment and longer lives with less disability.

In addition, Kurtzke quantified an MS disability scale that has evolved into the current Expanded Disability Status Scale (EDSS). The aim of the EDSS was to create an objective measure of the level of functioning that could be widely used by healthcare providers diagnosing, treating, and managing MS. The EDSS determines an overall score on a scale with a range from 0 to 10 . Lower EDSS scores, 0-4.5, denote patients with ambulatory capabilities, whereas higher EDSS scores, 5.0-9.5, identify MS patients with reduced ambulatory function [12]. EDSS is widely used in MS clinical trials, but not in daily clinical practices [24].

The purpose of this study was to map the components of the KFSS to ICD-9-CM codes to generate EDSS scores and calculate the change in EDSS scores, thus creating a tool that can identify MS patients with disability progression and quantify MS disability using administrative claims $[9,14]$.

The ethical nature of this trial was approved by the Intermountain Health Investigational Review Board, reference number 1050287. Patient consent was waived, as patient information was aggregated. Authors complied with all ethics guidelines. Furthermore, the Intermountain Health gave the appropriate permissions for use of their data, and their employees participated in the research and authoring of this manuscript. 


\section{METHODS}

\section{Study Design}

This study identified a retrospective cohort of MS patients from the Intermountain Healthcare Provider-Payer IDN [15] and mapped the ICD-9$\mathrm{CM}$ codes reported in their electronic health records (EHR) to KFSS components to derive an estimate of disability, the EDSS. The EDSS score assessed disability progression over the most recent 12-month care coverage period after index date, referred to as the "EDSS assessment period." The most recent 12-month care coverage period was selected as the "EDSS assessment period" for its compatibility with clinical trials as well as to capture the most current progression status of patients. Means, medians, ranges, and changes of KFSS and EDSS scores were calculated for this "EDSS assessment period."

\section{Data Sources}

\section{Intermountain Healthcare System}

All data for this study were sourced from the IDN Intermountain Healthcare database covering the period from January 1, 2010, to December 31, 2014. This database includes over 5 million patients across 22 hospitals, 185 clinics, and 750 physicians in Utah and Idaho. The EHR within the IDN Intermountain Healthcare archive is likely to capture all patient experiences within the network. Data within the EHR include inpatient and outpatient encounters, diagnoses, procedures, medication orders, laboratory results, clinical notes, and other information and associate these data with a single, unique identifier for each patient.

\section{KFSS and EDSS}

The KFSS, developed for MS patients to measure demyelination impact on body systems, evaluates eight systems. The KFSS component includes systems related to the brain (pyramidal, cerebellar, brainstem, sensory, and cerebral) as well as three body functioning systems: bowel and bladder, visual, and other [14].
The EDSS, Kurtzke's subsequent MS disability scale, is a 10-point scale that objectively quantifies disability and level of functioning. The EDSS calculates a total score on a scale that ranges from 0 to 10 . The first levels, $0-4.5$, refer to people with a greater degree of ambulatory ability, and the subsequent levels 5.0-9.5 refer to the impairment of ambulatory ability [14]. For this study's disability evaluation, the EDSS was adapted from the standard clinical EDSS. For example, the criterion EDSS score $=6$ was originally defined as "Intermittent or unilateral constant assistance (cane, crutch, brace) required to walk about $100 \mathrm{~m}$ with or without resting (usual functional systems [FS] equivalents are combinations with more than two FS grade $3+$ )." The modified EDSS score $=6$ was defined as (three FS grade $3+$; others FS grades $\leq 3$ ) or (two FS grade $4+$; others FS grades $\leq 3$ ). More details of the modified EDSS are presented in Table 1.

\section{Patients}

The progressive MS patient cohort was selected using a sequence of queries of the structured healthcare claims data. Patients with a diagnosis of MS, who were at least 18 years old, had 12 months of history in the database prior to the index date, had no documented pregnancy during the study period (or the 12-month preindex period), and who had no other demyelinating diseases were included [15]. The progressive MS cohort here included both primary progressive and secondary progressive MS (SPMS) patients. The SPMS diagnosis gradually develops from a relapsed-remitting multiple sclerosis (RRMS) diagnosis. Per literature, approximately $15 \%$ MS cases are diagnosed as having primary progressive MS $[1,2]$.

\section{Mapping}

First, ICD-9-CM codes of the MS patients were mapped to KFSS components. Then, the mapped KFSS components were further mapped to the modified EDSS; see Fig. 1. 
Table 1 Kurtzke Expanded Disability Status Scale (EDSS)

Score Evaluation

Clinical EDSS [14] Modified EDSS $^{\mathbf{b}}$

$0.0 \quad$ Normal neurologic examination (all grade 0 in all functional system [FS] scores $^{\mathrm{a}}$ )

1.0 No disability, minimal signs in one $\mathrm{FS}^{\text {a }}$ (i.e., grade 1)

All grade 0 in all functional system (FS) scores ${ }^{a}$

1.5 No disability, minimal signs in more than one $\mathrm{FS}^{\mathrm{a}}$ (more

One FS grade $1^{\text {a }}$ than 1 FS grade 1)

2.0 Minimal disability in one FS (one FS grade 2, others 0 or 1)

2.5 Minimal disability in two FS (two FS grade 2, others 0 or 1)

3.0 Moderate disability in one FS (one FS grade 3, others 0 or 1) or mild disability in three or four FS (three or four FS grade 2, others 0 or 1 ) though fully ambulatory

3.5 Fully ambulatory but with moderate disability in one FS (one grade 3 ) and one or two FS grade 2 or two FS grade 3 (others 0 or 1 ) or five grade 2 (others 0 or 1 )

4.0 Fully ambulatory without aid, self-sufficient, up and about some $12 \mathrm{~h}$ a day despite relatively severe disability consisting of one FS grade 4 (others 0 or 1 ) or combination of lesser grades exceeding limits of previous steps; able to walk without aid or rest $>500 \mathrm{~m}$

4.5 Fully ambulatory without aid, up and about much of the day, able to work a full day, may otherwise have some limitation of full activity or require minimal assistance; characterized by relatively severe disability usually consisting of one FS grade 4 (others or 1) or combinations of lesser grades exceeding limits of previous steps; able to walk without aid or rest $>300 \mathrm{~m}$

5.0 Ambulatory without aid or rest for about $200 \mathrm{~m}$; disability severe enough to impair full daily activities (e.g., to work a full day without special provisions) (usual FS equivalents are one grade 5 alone, others 0 or 1; or combinations of lesser grades usually exceeding specifications for step 4.0

(One grade 5 alone, others 0 or 1); or (two grade 4 and others $<3$ ) 
Table 1 continued

Score Evaluation

Clinical EDSS [14]

5.5 Ambulatory without aid for about $100 \mathrm{~m}$; disability severe enough to preclude full daily activities (usual FS equivalents are one grade 5 alone, others 0 or 1 ; or combination of lesser grades usually exceeding those for step 4.0)

6.0 Intermittent or unilateral constant assistance (cane, crutch, brace) required to walk about $100 \mathrm{~m}$ with or without resting (usual FS equivalents are combinations with more than two FS grade $3+$ )

6.5 Constant bilateral assistance (canes, crutches, braces) required to walk about $20 \mathrm{~m}$ without resting (Usual FS equivalents are combinations with more than two FS grade $3+$ )

7.0 Unable to walk beyond approximately $5 \mathrm{~m}$ even with aid, essentially restricted to wheelchair; wheels self in standard wheelchair and transfers alone; up and about in wheelchair some $12 \mathrm{~h}$ a day (usual FS equivalents are combinations with more than one FS grade $4+$; very rarely pyramidal grade 5 alone)

7.5 Unable to take more than a few steps; restricted to wheelchair; may need aid in transfer; wheels self but cannot carry on in standard wheelchair a full day; may require motorized wheelchair (usual FS equivalents are combinations with more than one FS grade $4+$ )

8.0 Essentially restricted to bed or chair or perambulated in wheelchair but may be out of bed itself much of the day; retains many self-care functions; generally has effective use of arms (usual FS equivalents are combinations, generally grade $4+$ in several systems)

8.5 Essentially restricted to bed much of day; has some effective use of $\operatorname{arm}(\mathrm{s})$; retains some self-care functions (usual FS equivalents are combinations, generally $4+$ in several systems)

9.0 Helpless bed patient; can communicate and eat (usual FS equivalents are combinations, mostly grade $4+$ )

9.5 Totally helpless bed patient; unable to communicate effectively or eat/swallow (usual FS equivalents are combinations, almost all grade $4+$ )

\section{Modified EDSS $^{\mathbf{b}}$}

One FS grade $5+$; others FS grades $\leq 3$

(Three FS grade $3+$; others FS grades $\leq 3$ ) or (two FS grade $4+$; others $\mathrm{FS}$ grades $\leq 3$ )

Three FS grade 3 + with pyramidal function grade 4; others FS grades $<3$

Two FS grade $4+$ with pyramidal function grade $4+$; others FS grades $<4$

Three FS grade $4+$ with pyramidal function grade $4+$; others FS grades $<4$

Four FS grade $4+$ with pyramidal function grade 6 ; others FS grades $<4$

Five FS grade $4+$ with pyramidal function grade 6 ; others FS grades $<4$

Six FS grade $4+$ with pyramidal function grade 6 ; others FS grades $<4$

All FS grade $4+$ with pyramidal function grade 6 
Table 1 continued

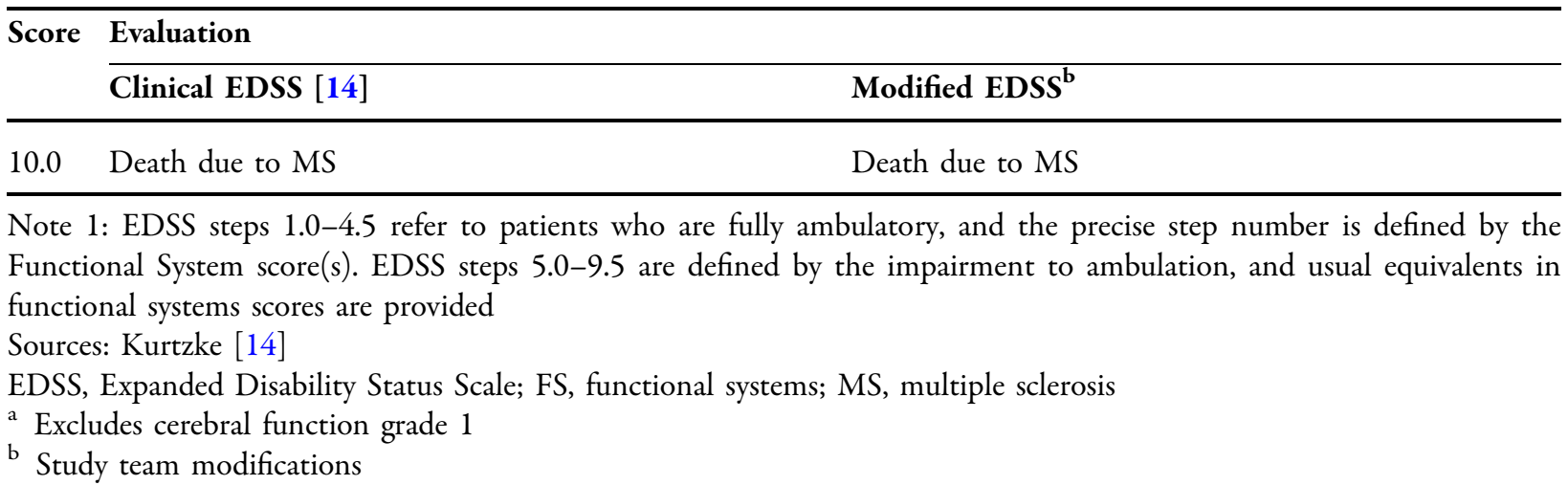

\section{KFSS}

As ICD-9-CM does not have specific codes aligned to KFSS signs and symptoms, crossmapping was performed. Two experienced clinicians linked individual code values to KFSS criteria, and two clinical informaticists reviewed how closely the terms were aligned. For example, for the pyramidal function system, motor functions, such as walking, were mapped as follows:

1-Abnormal signs without disability (ICD9-CM 781.2 abnormality of gait)

2-Minimal disability (ICD-9-CM 719.7, difficulty in walking)

6-Quadriplegia (ICD-9-CM: 344.00 quadriplegia unspecified, 344.09 other quadriplegia, and V49.84 bed confinement status), etc.

During the mapping, the KFSS 'Unknown' (value $=9$ ) was excluded and not mapped. The KFSS 'Normal' (value =0) was defined by an absence of an MS sign or symptom. Details of conversion from KFSS into ICD-9-CM codes are at Appendix 1 (Table 1 Supplementary).

\section{EDSS}

The EDSS score is based on a combination of the number of non-zero functional system grades and the actual grades based on the mapped KFSS. EDSS was mapped from the KFSS as shown in Table 1. Following mapping, the EDSS was modified for two reasons: (1) the lack of clinical detail regarding ambulatory function in ICD-9CM codes for mapping to KFSS; (2) it represents mutually exclusive EDSS categories (e.g., EDSS 6.0 Intermittent or unilateral constant assistance [cane, crutch, brace] required to walk about $100 \mathrm{~m}$ with or without resting); see Table 1. As reported in the literature, the lower scale values (0-4.0) were influenced by impairments detected by the neurologic examination of eight functional systems, while the values $>$ 4.0 are mainly based on the walking ability, and values $>6$ are based primarily on patients' handicaps $[5,25]$. Disability progression was defined by change between the first modified EDSS score during the first 7 to 12 months and the second modified EDSS score during the 1st-6th months of the "EDSS assessment period;" see Fig. 2. The rates of EDSS change varied depending on the initial values $[10,23]$. Change was required to be $\geq 1.0$ point if the first modified EDSS score was between 0 and 5.5 inclusive or $\geq 0.5$ point if the first modified EDSS score was $>5.5$. The guidelines of the European Medicines Agency (EMA) suggest that an average change from baseline is not an adequate efficacy parameter. Instead, they recommend defining treatment success or treatment failure of either reaching a certain EDSS score or a sustained change in sufficient volume [21]. A separate consideration of the lower and upper value range of the EDSS is recommended: a 1.0 point on the EDSS scale with a baseline EDSS score $\leq 5.5$ and 0.5 points in a baseline EDSS score $>5.5$ [6]. Six-month confirmed disability progression was defined as: 


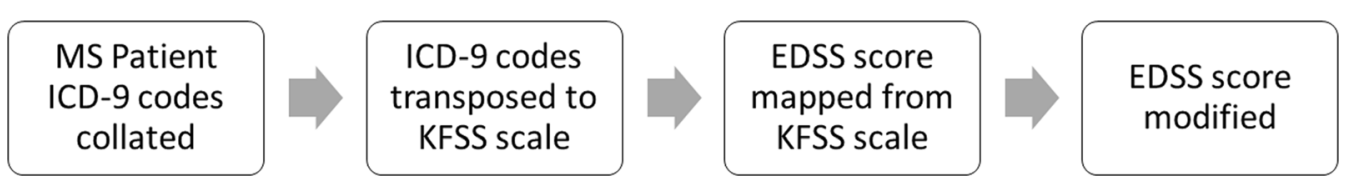

Fig. 1 Mapping flowchart. EDSS, expanded Disability Status Scale; ICD-9 International Classification of Diseases, 9th revision; KFSS, Kurtzke Functional Systems Score; MS, multiple sclerosis

- A sustained increase of 1 point on the EDSS for patients with baseline EDSS $\leq 5.5$ persisting for at least 6 months;

- A sustained increase of 0.5 for a patient with baseline EDSS $>5.5$, persisting for at least 6 months [18].

Previous studies showed that the rates of change varied depending on the initial value. Greater rates of change were observed at the lower end of the scale, which described less severe MS. However, for patients with more severe disease, with EDSS scores $\geq 6$, the EDSS showed very little change in sensitivity [5]. Ravnborg and colleagues reported higher annual rates of change (worsening health) at low EDSS baseline [23].

\section{RESULTS}

From a cohort of 2960 MS patients [15], 608 $(20.5 \%)$ were identified as experiencing progressive MS by change in modified EDSS score; see Table 2 and Appendix 2. Among these 608 patients, the median (range) first and second EDSS scores were $0(0-6)$ and $5(1-8)$, respectively. The median (range) change from the first to second EDSS score was 5 ( 1 to 7.5 ). The median first KFSS score for all systems was 0 . The mean first KFSS score varied by system, with the highest score (1.06) for sensory function and the lowest score $(0.12)$ for cerebellar functions; see Fig. 3.

Among 544 patients with first EDSS score in the group $\leq 2.5,9.7 \%$ still had the second EDSS score in the same group, and $75.2 \%$ and $15.1 \%$ of patients had the second EDSS score in group 3-5.5 and $\geq 6$, respectively. Among 62 patients with first EDSS score in the group 3-5.5, 41.9\% still had the second EDSS score in the same group, and $58.1 \%$ had the second EDSS score in group $\geq 6 ;$ see Table 2 . More details of distributions by the first and second EDSS score groups are presented in Appendix 2 (Table 2 Supplementary).

\section{DISCUSSION}

The potential of Real-World Data healthcare claims databases to yield a more thorough understanding of clinical conditions and treatments than has previously been available is staggering. To date, IDN resources have not harnessed ICD-9-CM codes to determine MS disability estimates. The ground-breaking mapping techniques presented here represent a promising, innovative, and affordable method for estimating MS disability.

These MS disability estimates are derived from real-world data. Healthcare providers and their associates with knowledge of disease states entered patient data into the EHR. For example, neurologic diagnoses may come from the HCP or a neurologist; a vision diagnosis would originate from the HCP or the patient's ophthalmologist. For the EHR system to achieve its objectives of providing a comprehensive view of patients' medical history, assisting clinical decision making, and improving patient outcomes, the Intermountain Healthcare EHR system is designed to support the entry of accurate and timely data (e.g., ICD-9-CM diagnosis codes, procedure codes, and clinical notes) [13]. However, there is a low possibility that the EHR contains incorrectly identified MS ICD-9-CM codes, and the same examiner reviewed at the two different time points.

While use of ICD-9-CM codes was novel, there is precedence for a real-world evidence (RWE) evaluation of EDSS to measure the disease progression in relapsing-remitting MS patients: a German NeuroTransData (NTD) MS registry study [3] that evaluated time to 3- and 
1/1/2012

Start of most recent

care coverage period

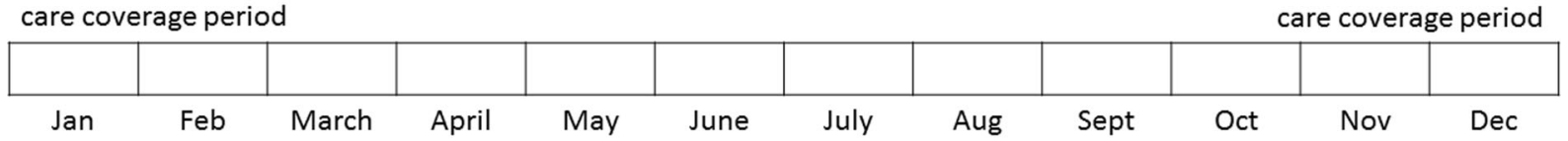

$12^{\text {th }}$ Month

$1^{\text {st }}$ Month

Fig. 2 An illustration of the most recent 12-month care coverage period after index date-the "EDSS assessment period"

Table 2 Distribution by first and second EDSS score groups during the EDSS assessment period among 608 progressive MS

\begin{tabular}{|c|c|c|c|c|c|c|c|}
\hline \multirow[t]{4}{*}{ First EDSS score group } & \multicolumn{7}{|c|}{ Second EDSS score group, $n$ (row \%) } \\
\hline & \multicolumn{6}{|c|}{ Severity } & \multirow[t]{3}{*}{ Total } \\
\hline & \multicolumn{2}{|c|}{$\begin{array}{l}\text { Mild } \\
\leq 2.5\end{array}$} & \multicolumn{2}{|c|}{$\begin{array}{l}\text { Moderate } \\
3-5.5\end{array}$} & \multicolumn{2}{|c|}{$\begin{array}{l}\text { Severe } \\
\geq 6\end{array}$} & \\
\hline & $\bar{n}$ & $\%$ & $\bar{n}$ & $\%$ & $\bar{n}$ & $\%$ & \\
\hline$\leq 2.5$ & 53 & 9.7 & 409 & 75.2 & 82 & 15.1 & 544 \\
\hline $3-5.5$ & 0 & 0.0 & 26 & 41.9 & 36 & 58.1 & 62 \\
\hline$\geq 6$ & 0 & 0.0 & 0 & 0.0 & 2 & 100.0 & 2 \\
\hline
\end{tabular}

EDSS, Expanded Disability Status Scale; MS, multiple sclerosis

6-month EDSS confirmed disability progression (CDP) as an exploratory analysis. Data from that German MS registry study included patients aged $\geq 18$ years with RRMS, $\geq 1$ relapse, and EDSS assessment(s). The current study defined the most recent 12-month care coverage period as its "EDSS assessment period" to be compatible with clinical trials and capture patients' most updated progression status, keeping in mind that the selected time period under study may have been too short to define and distinguish a patient's secondary progressive disease course.

This study seemed to demonstrate a superior diagnosis and documentation of symptoms for the sensory functional system compared to the other functional systems; the sensory score was the maximum KFSS score. As reported in the literature, the lower scale values (0-4.0) are influenced by impairments detected by the neurologic examination of eight functional systems. The mapped EDSS tool may less accurately reflect the functional system for EDSS score $>4.0$ mainly based on the walking ability and $>6$ mainly on patient disabilities $[5,23]$. In addition, as the EDSS estimation of MS disability is based primarily on ambulation and not on cognition and/or fatigue, this analysis may underestimate mild cases and low EDSS scores.

However, the changes of EDSS score observed in this study are consistent with previous studies [6], but inconsistent with others [7]. It is well known from other studies that the EDSS is heavily biased to locomotor function $[11,27]$. Several factors influence the EDSS changes, including the baseline EDSS score of the study population, disease duration, followup period, age of MS onset, primary or secondary progressive MS, and disease progression with or without relapse. In addition, clinical signs and/or symptoms documented via ICD-9CM codes in the EHR database for mild and moderate disability severity of MS were underrecorded and under-reported. 


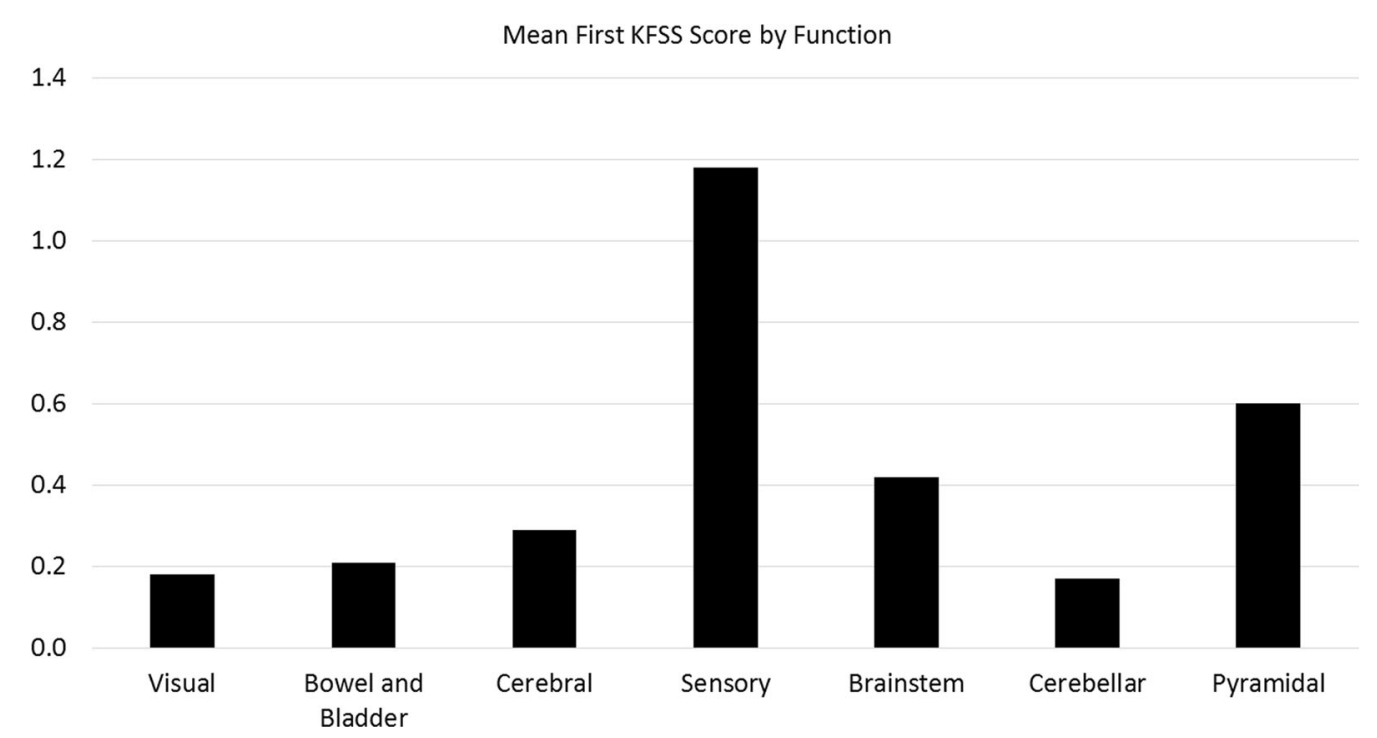

Fig. 3 Mean first KFSS score by function among 608 progressive MS patients during the “EDSS assessment period.” KFSS, Kurtzke Functional Systems Score

In the event of missing codes for KFSS signs and symptoms, this study assumed their absence. This assumption may have led to an overestimation of the change in modified EDSS between the first and second EDSS scores. Because this mapping algorithm was used to identify progressive MS patients to exclude part of a larger study, the exclusion of some relapse patients with increase in EDSS, which was not due to actual disability progression, was considered acceptable as part of the sensitive exclusion criteria. This should considered for potential implications when using this mapping algorithm, and users of this method should be aware of the limitation when applying for another study purpose.

Lack of an ICD-9-CM diagnosis code corresponding to a KFSS score may not have indicated the absence of a condition due to how data are collected and prioritization of diagnoses for reimbursement (e.g., more severe problems may have been recorded, or less severe conditions not considered relevant to the visit may not have been recorded). Hence, the KFSS mapping may be more likely to capture more severe conditions and underscore patients with less disability. A full validation was not performed on the KFSS mapping to assess performance and identify areas (e.g., tails of the scores) in which the mapping algorithms may differ.

In addition, the mapping to the EDSS score without the ambulatory clinical detail may have resulted in different scoring than that provided by the healthcare provider/neurology specialist. This difference may have led to misclassification bias, which will need to be considered by future study teams to assess the validity of the developed mapping algorithms and to improve interpretation of the study results for persons with moderate or severe MS.

Furthermore, disability progression from relapses versus progression independent of relapses could not be distinguished in this study. RRMS usually has progressive clinical disability, with or without superimposed relapses and remissions. Some other MS patients have progressive disease from the beginning, although there may be superimposed relapses and remissions. Neurologic disability may result from progression of the disease, relapses with incomplete remissions, or both $[4,17,26]$. As such, the change in EDSS score could be due to a relapse during the final 1 year of the patients' coverage and not indicate disability progression, introducing misclassification bias.

The claims-based KFSS mapping tool presented here is one of several newly developed 
MS disability tools derived from registry data available for clinical practice; other tools include the Novartis MSProDiscuss ${ }^{\mathrm{TM}}$, Genetech's smartphone app, FLOODLIGHT, and the BeCare MS App [8, 16, 19].

Future research to expand on this technique should include two validation studies. The first study is recommended to validate the developed mapping algorithm based on EDSS records. The second validation study is recommended to confirm the identified progressive MS cases using manual medical chart reviews. Additionally, the authors encourage this MS mapping algorithm be assessed in other US and non-US healthcare databases.

\section{CONCLUSIONS}

This MS mapping study established that using mapping techniques to correlate ICD-9-CM codes to KFSS components was a productive use of EHR data. In addition, this analysis demonstrated that mapping of KFSS using ICD-9-CM makes possible the calculation of change in EDSS score and a proxy of MS disability progression.

Furthermore, this analysis contributes to the toolbox utilizing real-world data to calculate an MS patient clinical quality measure, which can support assessment of patient care and signal disability progression.

The results showed progressive MS patients had a wide range of EDSS score changes during their last year of care coverage. The developed mapping techniques will be a promising method for future comparative effectiveness and safety research of DMTs in real-world data databases.

\section{ACKNOWLEDGEMENTS}

The authors thank Amanda Shields, PAREXEL, Chapel Hill, NC, USA, and H.R. Crooke, former employee of PAREXEL, Durham, NC, USA, for their contributions to the manuscript.
Funding. This study was funded by EMD Serono (CrossRef Funder ID: https://doi.org/10. 13039/100004755). The Advances in Therapy Rapid Service and Open Access fees have been funded by the healthcare business of Merck KGaA, Darmstadt, Germany (CrossRef Funder ID: https://doi.org/10.13039/100009945).

Medical Writing Support. Medical writing was provided by Catherine Lareau of Lareau Consulting LLC., USA, and was supported by the healthcare business of Merck KGaA, Darmstadt, Germany (CrossRef Funder ID: https:// doi.org/10.13039/100009945).

Authorship. All named authors meet the International Committee of Medical Journal Editors (ICMJE) criteria for authorship for this article, take responsibility for the integrity of the work as a whole, and have given their approval for this version to be published.

Authors' contributions. Hoa V. Le and Chi T.L. Truong determined the study design, analyzed and interpreted results of the Intermountain Health patient data, and were major contributors to writing the manuscript. Aaron W.C. Kamauu interpreted results and was a contributor in writing the manuscript. John R. Holmen analyzed and was a contributor in writing the manuscript. Christopher L. Fillmore analyzed and was a contributor in writing the manuscript. Monica G. Kobayashi interpreted results and was a contributor in writing the manuscript. Schiffon L. Wong consulted on the study design, interpreted results and was a major contributor in writing the manuscript. All authors read and approved the final manuscript.

Disclosures. Chi T.L. Truong is an employee of MedCodeWorld. Hoa V. Le was an employee of PAREXEL and a stockholder of GlaxoSmithKline and was a Harry Guess-Merck merit scholarship recipient. Aaron W.C. Kamauu works for Ikaika Health and was president of Anolinx, LLC, at the time of the analyses; he received a research grant from the healthcare business of Merck KGaA, Darmstadt, Germany. John R. Holmen has nothing to disclose. 
Christopher L. Fillmore has nothing to disclose. Monica G. Kobayashi is an employee and stockholder of GlaxoSmithKline $(<1 \%)$. Schiffon L. Wong is an employee EMD Serono, Billerica, MA, USA.

Compliance with Ethics Guidelines. The ethical nature of this trial was approved by the Intermountain Health Investigational Review Board, reference number 1050287. Patient consent was waived, as patient information was aggregated. Authors complied with all ethics guidelines. Furthermore, Intermountain Health gave the appropriate permissions for use of their data, and their employees participated in the research and authoring of this manuscript.

Data Availability. Appropriate medical coding lists used for the study and manuscript are available upon request. However, patient data are not available due to HIPPA and privacy.

Open Access. This article is licensed under a Creative Commons Attribution-NonCommercial 4.0 International License, which permits any non-commercial use, sharing, adaptation, distribution and reproduction in any medium or format, as long as you give appropriate credit to the original author(s) and the source, provide a link to the Creative Commons licence, and indicate if changes were made. The images or other third party material in this article are included in the article's Creative Commons licence, unless indicated otherwise in a credit line to the material. If material is not included in the article's Creative Commons licence and your intended use is not permitted by statutory regulation or exceeds the permitted use, you will need to obtain permission directly from the copyright holder. To view a copy of this licence, visit http://creativecommons.org/licenses/bync/4.0/.

\section{REFERENCES}

1. Andersson PB, WE. Multiple sclerosis that is progressive from the time of onset: clinical characteristics and progression of disability. Arch Neurol. 1999;1138-1142.
2. Antel J, Antel S. Primary progressive multiple sclerosis: part of the MS disease spectrum or separate disease entity? Acta Neuropathol. 2012;123(5):627-38.

3. Braune SG. Comparative effectiveness of delayedrelease dimethyl fumarate versus interferon, glatiramer acetate, teriflunomide, or fingolimod: results from the German NeuroTransData registry. J Neurol. 2018;265:2980.

4. Confavreux C, Aimard G. Course and prognosis of multiple sclerosis assessed by the computerized data processing of 349 patients. Brain. 1980;103:281-300.

5. Drulovic J, Riise T. Self-rated physical health predicts change in disability in multiple sclerosis. Mult Scler. 2008;14:999-1002.

6. (EMA) EM guideline of clinical investigation of medical products for the treatment of multiple sclerosis. Draft. EMA/ CHMP/771815/2011, Rev 2. 2012. http://www.ema.europa.eu/docs/en_GB/ document_library/Scientific_guideline/2012/10/ WC500133438.pdf. Accessed 21 Jan 2021.

7. Fernández O, Oreja-Guevara C. Natalizumab treatment of multiple sclerosis in Spain: results of an extensive observational study. J Neurol. 2012;259(9):1814-23.

8. Floodlight Open. 2021. https://floodlightopen. com/en-US. Accessed 13 May 2021.

9. Gajofatto AA. Treatment strategies for multiple sclerosis: when to start, when to change, when to stop? World J Clin Cases. 2015;3:545-55.

10. Hohol MJ, Orav EJ. Disease steps in multiple sclerosis: a longitudinal study comparing disease steps and EDSS to evaluate disease progression. Mult Scler. 1999;5:349-54.

11. Hoogervorst ELJ, Eikelenboom MJ. One year changes in disability in multiple sclerosis: neurological examination compared with patient self report. J Neurol Neurosurg Psychiatry 2003;74:439-42.

12. Hughes BKM. Breaking new ground with RWE: how some pharmacos are poised to realize a $\$ 1$ billion opportunity. IMS Health White Paper. 2014. https://www.iqvia.com/-/media/quintilesims/pdfs/ qims_breaking_new_ground_with_rwe_whitepaper. pdf. Accessed 13 May 2021.

13. Intermountain Healthcare. 2021. https://inter mountainhealthcare.org/-/media/files/trustee-resou rce-center/topical-information-pdfs/electronic-heal th-record.pdf?la=en. Accessed 13 May 2021.

14. Kurtzke J. Rating neurologic impairment in multiple sclerosis: an expanded disability status scale (EDSS). Neurology. 1983;33:1444-52. 
15. Le HV, Le Truong CT. Identifying patients with relapsing-remitting multiple sclerosis using algorithms applied to US integrated delivery network healthcare data. Value Health. 2019;22:77-84.

16. Litchman C, Stoll S. Automated EDSS scoring using a mobile app and machine learning. Neurology. 2020;94(15 Supplement):2631.

17. Lublin FD, Reingold SC. Defining the clinical course of multiple sclerosis: results of an international survey. Neurology. 1996;46:907-11.

18. Meyer-Moock S, Feng YS. Systematic literature review and validity evaluation of the Expanded Disability Status Scale (EDSS) and the Multiple Sclerosis Functional Composite (MSFC) in patients with multiple sclerosis. BMC NNeurol. 2014;14:58.

19. MS ProDiscuss. 2021. https://www.msprodiscuss. com/. Accessed 13 May 2021.

20. Sharrack B, Hughes RA. Clinical scales for multiple sclerosis. J Neurol Sci. 1996;135:1-9.

21. Ravnborg M, Blinkenberg M. Responsiveness of the multiple sclerosis impairment scale in comparison with the expanded disability status scale. Mult Scler. 2005;11:81-4.
22. Ronicke VRM. Revitalizing pharmaceutical R\&D. The value of real word evidence. Strategy \&. PWC White Paper. 2015. https://www.strategyand.pwc. com/gx/en/insights/2015/revitalizing-pharmaceuti cal-rd.html. Accessed 21 Jan 2021.

23. US Department of Veteran Affairs MS. Kurtzke Expanded Disability Status Scale. 2021. https:// www.va.gov/MS/Professionals/diagnosis/Kurtzke_ Expanded_Disability_Status_Scale.asp. Accessed 21 Jan 2021.

24. van Munster CE, Uitdehaag BMJ. Outcome measures in clinical trials for multiple sclerosis. CNS Drugs. 2017;31:217-36.

25. Vaney C, Blaurock H. Assessing mobility in multiple sclerosis using the rivermead mobility index and gait speed. Clin Rehabil. 1996;10:216-26.

26. Weinshenker BG, Bass B. The natural history of multiple sclerosis: a geographically based study I Clinical course and disability. Brain. 1989;112: 133-46.

27. Whitaker JN, McFarland HF. Outcomes assessment in multiple sclerosis clinical trials: a critical analysis. Mult Scler. 1995;1:37-47. 